\title{
Moderate-gain Brillouin amplification: An analytical solution below pump threshold
}

\author{
Fikri Serdar Gokhan \\ Department of Electrical and Electronics Engineering, Faculty of Engineering and Architecture, Gazikent University, 27410 Sahinbey, Gaziantep, Turkey
}

\section{A R T I C L E I N F O}

Article history:

Received 30 December 2010

Accepted 24 June 2011

Available online 13 July 2011

\section{Keywords:}

Scattering

Stimulated Brillouin

Fiber optics amplifiers and oscillators

Nonlinear optics

Fibers

\begin{abstract}
A B S T R A C T
Highly accurate closed-form analytical formula, based on the undepleted pump approximation (UPA), is derived for pump, Stokes and the gain of a Brillouin fiber amplifier (BFA) operating in the moderately depleted pump regime. The material loss of the medium, which is a strong effect in optical fibers, is taken into account. To define validity ranges of the solution, the threshold power of BFA is accurately determined. The obtained results are numerically and experimentally validated. The presented analysis can be used to accurately predict the performance of moderate-gain BFAs.
\end{abstract}

(c) 2011 Elsevier B.V. All rights reserved.

\section{Introduction}

Stimulated Brillouin scattering (SBS) is the most efficient nonlinear amplification mechanism in optical fibers, in which a large gain may be obtained under the pump power of several milliwatts [1]. This has led to the design of BFA configuration and has been implemented in a wide range of applications, such as an active filter due to its narrowband amplification feature [2] or in the control of pulse propagation in optical fibers [3]. The BFA can also be used to measure strain and temperature [4] which has led to the design of distributed Brillouin sensors (DBS). In these types of sensors, strain and temperature can be measured along the whole fiber length. The performance of the BFA fiber sensors needs to account for pump depletion which can be investigated using the solution of the coupled intensity equations which describe the interaction between the pump and the Stokes wave due to SBS. The solution of the mentioned ordinary differential equations (ODEs) is socalled two-point boundary value problem comprising the initial values of the pump and the Stokes waves at input points. The exact solution to the problem is known only for lossless media [5]. However, this solution is not applicable to BFAs where wave interaction occurs over tens of kilometers and loss is a significant effect which will introduce much error into the sensing accuracy.

An approximate solution based on the UPA is accurate enough to answer many practical questions. However, the accuracy of UPA is not sufficient for the applications which call for the exact solution, e.g., Brillouin sensors. To improve the accuracy of the UPA solution, one can supply highly effective guess expression as a starting point for the determination of Stokes and thus pump wave. The accuracy of the UPA solution is proportional to the success of the intuitive guess expression.

E-mail address: fsgokhan@gmail.com.
Several attempts have been undertaken to find a general analytical solution to SBS equations in a lossy medium [6-9]. In Ref. [6], the system of ODEs is reduced to a single equation which can only be integrated numerically. In Ref. [7], the proposed solution results in a system of two transcendental equations to be solved numerically with no closed-form solution possible. In another approach [8], an approximate solution is presented but is only valid for moderate pump powers. Recently, an approximate analytic solution of the steady state coupled equations has been proposed [9]. However, these equations include the term of stokes intensity at the fiber input, which is not known prior to the computation.

In this paper, highly accurate UPA based solution of pump, Stokes and Gain of the BFA operating in the moderately depleted pump regime is introduced. Comparisons were made in terms of accuracy with the proposed solution and the UPA and worst-case analysis is carried out. The threshold power of BFAs is accurately determined by extending the expression proposed by the authors in Ref.[10]. The proposed solution is validated by making comparison with experimental data and full numerical integration of coupled ODEs. Validity ranges of the found solution are discussed. Simulation results show that, at the worst case, the absolute error of the proposed solution is 5 and 2.3 times better than the UPA solution, for pump and Stokes and respectively.

\section{Theoretical model}

The coupled ODEs for the evolution of the intensities of pump $I_{p}$ and Stokes $I_{S}$ can be written as [5]

$d I_{p} / d z=-g_{B} I_{P} I_{S}-\alpha I_{p}$

$d I_{S} / d z=-g_{B} I_{P} I_{S}+\alpha I_{S}$ 
where $0 \leq z \leq L$ is the propagation distance along the optical fiber of the total length $L, \alpha$ is the fiber loss coefficient, $g_{B}$ is the Brillouin gain coefficient. Here we assume Stokes wave launched from the rear end of the fiber. Then the known values of the input pump intensity $I_{p}(0)$ and the input Stokes intensity $I_{s}(L)$ are the boundary values. If the pump is attenuated by the fiber loss only, which is said to be undepleted, the pump dependence with its length is (UPA),

$I_{p}(z)=I_{p}(0) \cdot \exp (-\alpha \cdot z)$.

The stokes intensity is obtained by substitution of approximation Eq. (2) into Eq. (1b) with subsequent integration over the whole fiber length of the latter results in,

$I_{S}(0)=I_{S}(L) \cdot \exp \left(g_{B} \cdot I_{p}(0) \cdot L_{e f f(L)}-\alpha \cdot L\right)$

where $L_{e f f(x)}=\left(1-e^{-\alpha \cdot x}\right) / \alpha$ is the effective length of distance $x$. Using Eq. (1b), $I_{s}(z)$ can be obtained as,

$I_{s}(z)=I_{s}(0) \cdot \exp \left(\alpha \cdot z-g_{B} I_{p}(0) L_{e f f(z)}\right)$.

Substitution of Eq. (3) into Eq. (4), the known Stokes expression based on the UPA can be obtained as,

$I_{s}(z)=I_{S}(L) \cdot \exp \left(g_{B} I_{p}(0) \frac{e^{-\alpha L}}{\alpha}\left(e^{\alpha(L-z)}-1\right)-\alpha(L-z)\right)$

Solution of $I_{p}(z)$ can be obtained by Eq. (1a),

$I_{p}(z)=I_{p}(0) \exp \left\{-\alpha \cdot \int_{0}^{z} d z-\int_{0}^{z} g_{B} I_{S}(z) d z\right\}$

Substitution of Eq. (5) into Eq. (6), we get,

$I_{p}(z)=I_{p}(0) \exp \left\{-\alpha \cdot z+\int_{L}^{L-z} g_{B} I_{S}(L) \exp \left[g_{B} I_{p}(0) \frac{e^{-\alpha L}}{\alpha}\left(e^{\alpha \zeta}-1\right)-\alpha \zeta\right] d \zeta\right\}$

where,

$$
\begin{gathered}
\int_{L}^{L-z} g_{B} I_{S}(L) \exp \left[g_{B} I_{p}(0) \frac{e^{-\alpha L}}{\alpha}\left(e^{\alpha \zeta}-1\right)-\alpha \zeta\right] d \zeta \\
=-g_{B} \cdot I_{S}(L) \cdot C_{1} \cdot \exp \left(-C_{2} / \alpha\right) / \alpha^{2}
\end{gathered}
$$

The coefficients of Eq. (8) are as follows,

$C_{1}=\left(-\alpha \cdot \exp \left(\mathrm{g}_{B} \cdot I_{P}(0) / \alpha\right)-\mathrm{C}_{3} \cdot \mathrm{g}_{B} \cdot I_{P}(0)+\alpha \cdot \mathrm{C}_{4}+\mathrm{C}_{5} \cdot \mathrm{g}_{B} \cdot I_{P}(0)\right)(9)$

$C_{2}=\left(g_{B} \cdot I_{p}(0) \cdot \exp (-\alpha L)+\alpha^{2} L\right)$

$\mathrm{C}_{3}=\operatorname{Ei}\left(1,-\mathrm{g}_{B} \cdot I_{p}(0) / \alpha\right)$

$C_{4}=\exp \left(\left(\alpha^{2} z+g_{B} \cdot I_{p}(0) \cdot \exp (-\alpha \cdot z)\right) / \alpha\right)$

$\mathrm{C}_{5}=\operatorname{Ei}\left(1,-\exp (-\alpha \cdot \mathrm{z}) \cdot \mathrm{g}_{B} \cdot I_{p}(0) / \alpha\right)$.

In Eqs. (11) and (13), the Ei function implies as

$\operatorname{Ei}\{n, z\}=\int_{1}^{\infty} \frac{e^{-z t}}{t^{n}} d t \quad n \geq 0$
The terms $\operatorname{Ei}\left\{1,-g_{B} I_{p}(0) / \alpha\right\}$ and $\operatorname{Ei}\left(1,-\exp (-\alpha \cdot z) \cdot g_{B} \cdot I_{p}(0) / \alpha\right)$ can be evaluated using command line of Matlab ${ }^{\mathrm{TM}}$ as,

$m f u n\left({ }^{\prime} E i^{\prime}, 1,-\exp (-\alpha \cdot z) \cdot g_{B} I_{p}(0) / \alpha\right)$

mfun $\left({ }^{\prime} E i^{\prime}, 1,-g_{B} I_{p}(0) / \alpha\right)$.

Pump power evolution based on Eq. (7) is plotted in Fig. 1. As can be seen from the figure, the pump power is matching very well with the numerical solution up to the threshold level $\left(P_{T h}=3.3 \mathrm{~mW}\right)$. When the input pump power threshold is crossed, discrepancy appears. This is due to the fact that, the proposed analytical solution is based on the UPA which is started with the intuitive guess expression of Eq. (2).

Maximum error which occurs at the threshold pump power is $0.1 \%$ of the pump input power which indicates the excellent agreement with the exact numerical solution below threshold. It is seen in Fig. 1 (c) that, as the pump power decreases, error ratio of UPA/Proposed solution increases. For pump power of 1 and $3 \mathrm{~mW}$, the absolute error of the proposed solution is 687 and 9 times better than the UPA solution, respectively. At the worst case, which it occurs at the threshold power level, the error performance of the proposed solution is 5 times better than the UPA solution.
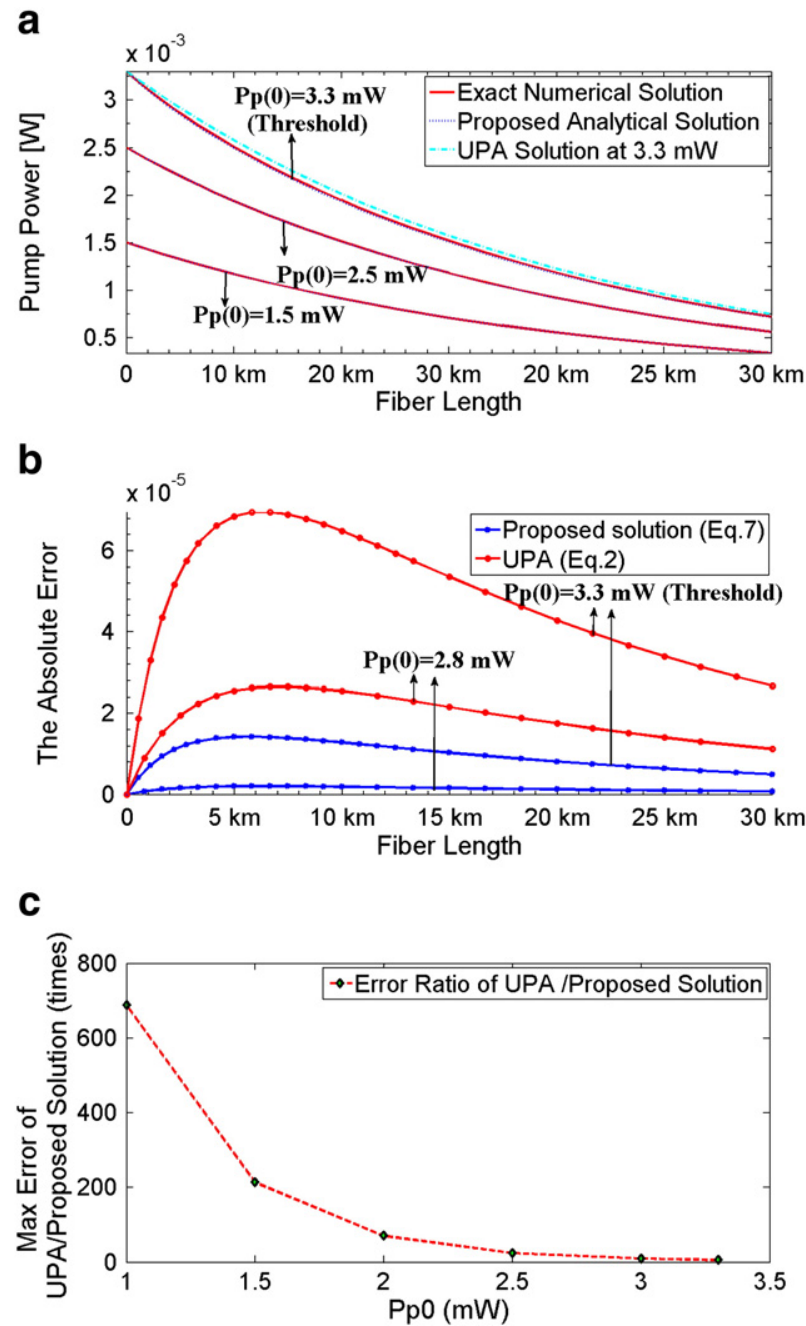

Fig. 1. (a) Distribution of pump power along the fiber using numerical solution and proposed analytical solution. $P_{p}(0)=1.5,2.5$, and $3.3 \mathrm{~mW}, P_{s}(L)=410 \mathrm{nW}$, $A_{\text {eff }}=80 \mu \mathrm{m}^{2}, \mathrm{~L}=30 \mathrm{~km}, \mathrm{~g}_{\mathrm{B}}=1.091 \times 10^{-11}, \alpha=0.215 \mathrm{~dB} / \mathrm{km}$ (b) The absolute error between numerical and proposed solution of Eq. (2) (UPA) and Eq. (7). (c) Maximum Error ratio of UPA and Proposed Solution (times). 
The value of the absolute error is an important criterion for the determination of the Stokes wave evolution. For example, although Eq. (15) seems to reflect correctly the behavior of the Stokes wave, its absolute error performance is only $8 \%$ better (at $P_{T h}$ ) than UPA solution (Eq. (5)) and this performance is not satisfactory beside its solution complexity. Details of the solution of Eq. (15) are given in the Appendix.

$I_{S}(z)=I_{S}(L) \exp \left\{\alpha \cdot(z-L)+\int_{L}^{z} g_{B} I_{p}(0) \exp \left[g_{B} I_{S}(L) \frac{e^{-\alpha L}}{\alpha}\left(e^{\alpha \zeta}-1\right)-\alpha \zeta\right] d \zeta\right\}$

To lessen absolute error, the derivation of the Stokes expression has to be started with more intuitive pump expression compared with Eq. (2). This can be achieved by using $I_{p}(L)$ obtained by using Eq. (7). Thus, by using $I_{p}(L), I_{p}(z)$ can be obtained as,

$I_{p}(z)=I_{p}(0) \cdot e^{-\alpha \cdot k \cdot z}$

for $z=L$,

$I_{p}(L)=I_{p}(0) e^{-\alpha \cdot k \cdot L}$

$=I_{p}(0) \exp \left\{-\alpha \cdot L-\int_{0}^{L} g_{B} I_{S}(L) \exp \left[g_{B} I_{p}(0) \frac{e^{-\alpha L}}{\alpha}\left(e^{\alpha \zeta}-1\right)-\alpha \zeta\right] d \zeta\right\}$

$k=-(1 /(\alpha \cdot L)) \cdot \ln \left(I_{p}(L) / I_{p}(0)\right)$.

Substitution of Eq. (18) into Eq. (16), and the latter into Eq. (1b), $I_{S}(z)$ can be obtained as,

$$
\begin{aligned}
I_{S}(z)= & I_{S}(0) / \exp \left(g_{B} I_{p}(0) / \alpha / k\right) \\
& \times \exp \left\{\left(g_{B} I_{p}(0) \cdot \exp (-\alpha \cdot k \cdot z)+\alpha^{2} \cdot z \cdot k\right) / \alpha / k\right\} .
\end{aligned}
$$

In Eq. (19), $I_{S}(0)$ can be obtained by using the boundary value at $z=L$, thus;

$$
\begin{aligned}
I_{S}(z)= & I_{S}(L) \cdot \exp \left\{\left\{g_{B} I_{p}(0) \cdot[\exp (-\alpha \cdot k \cdot z)-\exp (-\alpha \cdot k \cdot L)]\right.\right. \\
& \left.\left.+\alpha^{2} \cdot k \cdot(z-L)\right\} / \alpha / k\right\} \quad P_{p}(0) \leq P_{T h} .
\end{aligned}
$$

Stokes power evolution based on Eq. (20) is plotted in Fig. 2. As can be seen from the figure, the calculated output stokes power is matching very well with the numerical solution below threshold. As in the case of pump, discrepancy also appears for the Stokes power over the threshold level. As can be seen in Fig. 2(c), worst-case relative error is observed near threshold. For the worst case, maximum error of UPA is 2.3 times more than the maximum error of proposed Stokes equation (Eq. 20).

The BFA gain is defined as the ratio of the amplified Stokes power and the launched Stokes power $G_{B F A}=P_{S}(0) / P_{S}(L)$. It can be calculated from Eq. (20) by setting $z=0$, the gain can be expressed as,

$$
\begin{aligned}
G_{B F A} & =\frac{P_{S}(0)}{P_{S}(L)} \\
& =\exp \left\{\left\{g_{B} I_{p}(0) \cdot[1-\exp (-\alpha \cdot k \cdot L)]-\alpha^{2} \cdot k \cdot L\right\} / \alpha / k\right\} \quad P_{p}(0) \leq P_{T h} .
\end{aligned}
$$

Brillouin sensors call for the solution of the steady state equations (Eq. (1a-1b)). This set of equations is valid for pulses larger than phonon lifetime ( $\approx 10 \mathrm{~ns}$.), which is equivalent to a spatial resolution, $w>1 \mathrm{~m}$. If it is considered that gain is uniform over the spatial resolution; it can be integrated over $[z, z+w]$. The variation in the
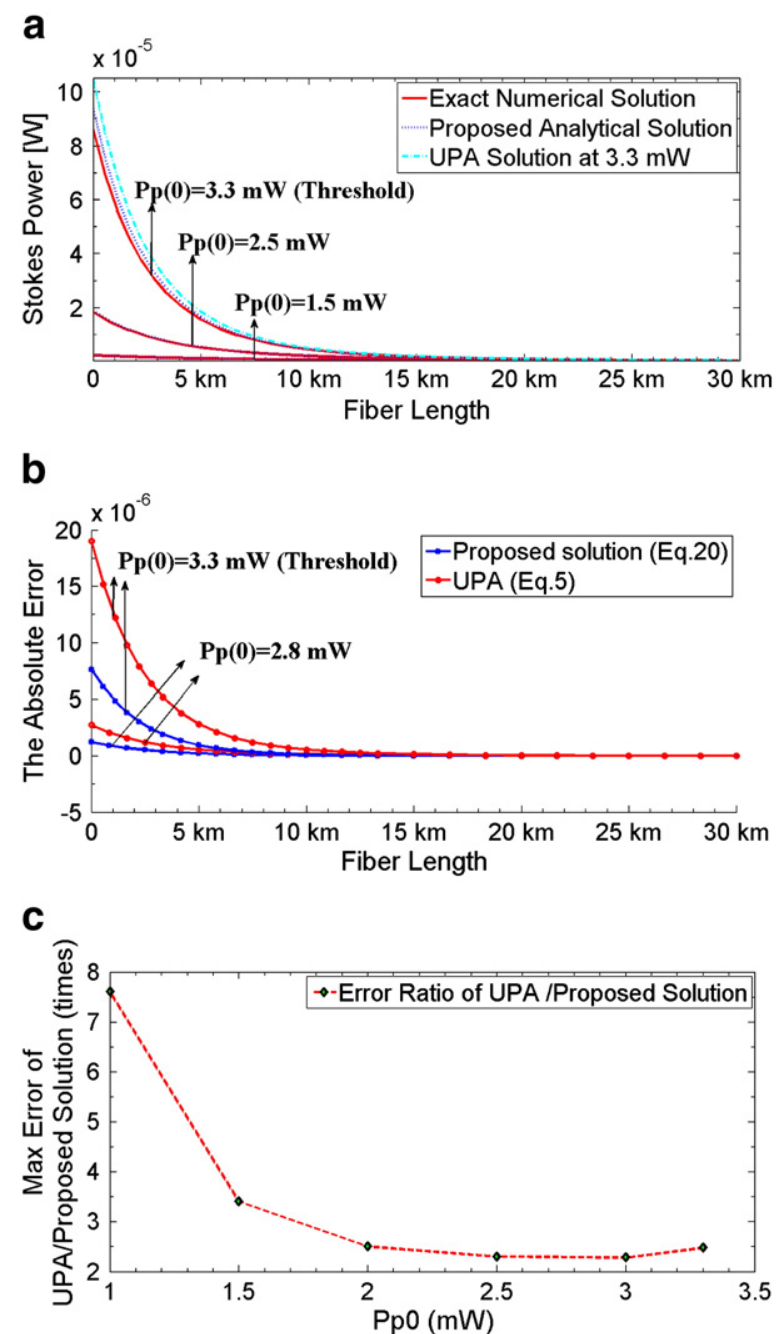

Fig. 2. (a) Distribution of Stokes power along the fiber using numerical solution and proposed analytical solution. $P_{p}(0)=1.5,2.5$ and $3.3 \mathrm{~mW}, P_{s}(L)=410 \mathrm{nW}$, $\mathrm{A}_{\text {eff }}=80 \mu \mathrm{m}^{2}, \mathrm{~L}=30 \mathrm{~km}, \mathrm{~g}_{\mathrm{B}}=1.091 \times 10^{-11}, \alpha=0.215 \mathrm{~dB} / \mathrm{km}$ (b) The absolute error between numerical and proposed solution of Eq. (5) (UPA) and Eq. (20). (c) Maximum Error ratio of UPA and Proposed Solution (times).

intensity of the cw signal due to the interaction with the pulse at position $z$ is given by [11],

$G=I_{s}(z) / I_{s}(z+w)$.

$G$ is known as the Brillouin loss amplitude, varies with the sensing fiber characteristics as $g\left(v_{\mathrm{B}}, \Delta v_{\mathrm{B}}\right), L, z$. The two beams involved in the sensing process have a measurable effect through $w, I_{p}$ and $I_{s}(L)$. To investigate the worst-case analysis, the relative error is introduced as,

$R E=\left|\left(I_{s}-I_{S_{-} \text {computed }}\right) / I_{S}\right|$.

The worst-case values of $R E$ occur for $z=0$ and $P \approx P_{T h}$. For the worst case, $R E$ is $8.8622 \%$ and $22.02 \%$ for the proposed solution and UPA, respectively. For $P \approx P_{T h} / 2$, at $z=0, R E$ is $0.2388 \%$ and $0.7340 \%$, and for $P \approx P_{T h} / 4, R E$ is $0.0065 \%$ and $0.1115 \%$ for the proposed solution and UPA, respectively. Obviously the error decreases for both, as $P$ decreases. 


\section{Determination of the threshold point of BFA}

In Ref. [10], for the Brillouin fiber generator (BFG), the authors investigated the Stokes backscattered $P_{s}(0)$ and transmitted $P_{p}(L)$ powers as well as the rate of change of the backscattered power against input pump power $d P_{s}(0) / d P_{p}(L)$. They observed that the inflection point of the $P_{s}(0)$ curve which occurs at the maximum of $d P_{s}$ $(0) / d P_{p}(L)$ corresponds to the threshold of BFG. Then, they have verified that the inflection point can also be used for the determination of BFA threshold. By following their arguments, the investigation of the derivative of transmitted powers of Stokes versus pump power $\left(d P_{s}(0) / d P_{p}(L)\right)$ is plotted in Fig. 3(a) and the threshold point is found as $3.3 \mathrm{~mW}$. The threshold level is verified in Fig. 3(b) and seen that after the threshold value, the error rate abruptly increases. Hence, for the worst case maximum error occurs at the threshold value. Maximum error at threshold power value of $P_{p}(L)$ and $P_{s}(0)$ is $0.7 \%$ and $8.8 \%$, respectively. These values imply that, proposed solutions of Eq. (7) and Eq. (20) can be considered as semi-exact analytical solutions below threshold.

For the determination of the input pump power threshold level, the analytical power threshold definition can be addressed [10],

$P_{t h}=\frac{A_{\text {eff }}}{\chi_{S B S} g_{B} L_{e f f}} \ln \left[\frac{\zeta A_{e f f}}{P_{S}(L) \chi_{S B S} g_{B} L_{e f f}}\right]$

where $A_{\text {eff }}$ is the fiber effective area and $\chi_{\mathrm{SBS}}$ is the depolarization factor. The authors found that $\zeta=0.97$. However, in order to improve accuracy, we have estimated $\zeta$ values by numerical fitting the maxima of $\left(d P_{s}(0) / d P_{p}(L)\right)$ to the analytical threshold definition in Eq. (22) and also experimentally verified as

$\zeta=2329 \times\left(P_{S}(L)\right)^{0.7763}+0.03762 \quad P_{S}(L)<60 \mu W$

$\zeta=2.04 \times 10^{4} \times P_{S}(L)+0.07633 \quad P_{S}(L)>60 \mu W$.

The coefficients of Eq. (23) and Eq. (24) are determined with a standard error of $R=0.9999$ and $R=0.9986$, respectively. The upper

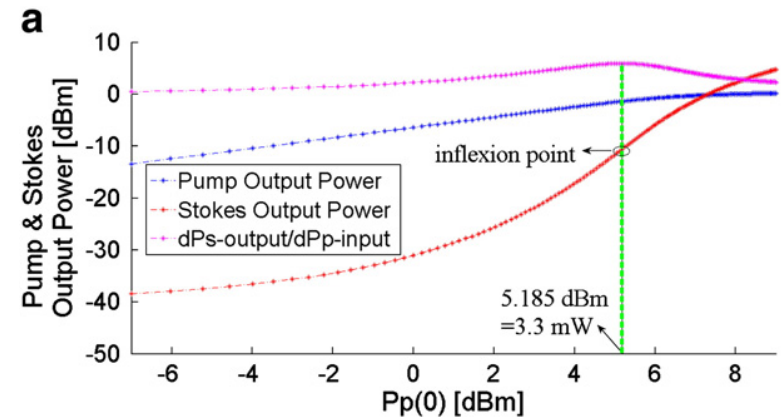

b

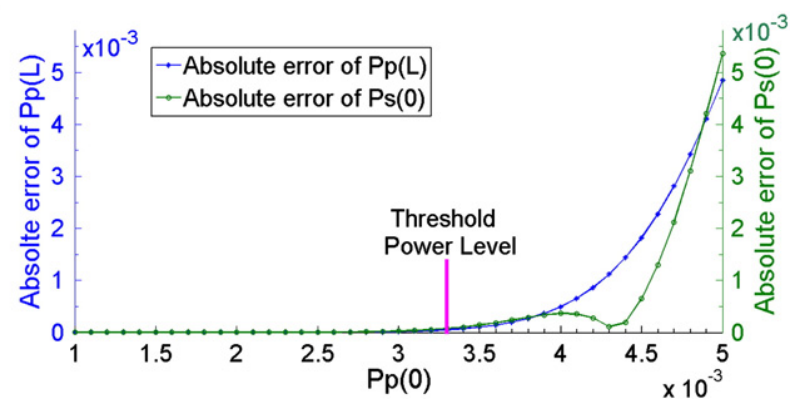

Fig. 3. (a) Derivative of Stokes backscattered power versus input pump $\left(d P_{s}(0) / d P_{p}(L)\right)$ for the scheme in Fig. 1 and 2. (b) Absolute error between numerical and proposed analytical solution of $P_{s}(0)$ and $P_{p}(L)$ vs. pump input power.

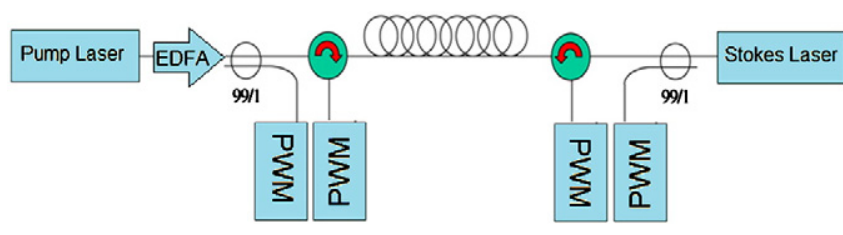

Fig. 4. Schematic of the experimental set-up.

value of $P_{S}(L)$ is $120 \mu \mathrm{W}$ for Eq. (24) and $\chi_{\text {SBS }}$ is $2 / 3$ for both Eq. (23) and Eq. (24).

To verify Eq. (20) we have performed measurements in a standard single-mode optical fiber using light from a tunable $1549.5 \mathrm{~nm}$ laser followed by an erbium-doped fiber amplifier as a pump. In our experimental set-up [Fig. 4], we measured the output Stokes wave $P_{s}$ $(0)$ at the pump input. Stokes and pump sources are Erbium doped fiber lasers, whose linewidths are smaller than $100 \mathrm{kHz}$. Their frequency difference is controlled with a phase locked loop and is locked to the Brillouin frequency, $v_{B}$ of the fiber under test. Power meters were used to monitor the input pump power $P_{p}(0)$, the transmitted pump power $P_{p}(L)$, the launched seed power $P_{s}(L)$, and the amplified Stokes power $P_{S}(0)$. A $30 \mathrm{~km}$ length of fiber was experimentally studied under these experimental conditions.

The experimental results are plotted in Fig. 5 together with threshold power level. As in Fig. 1, an increased discrepancy is observed for the transmitted pump power [Fig. 5(a)] in the regime of strong input pump over the $P_{T h}$. As for the amplifier gain $\left(G_{B F A}\right)$, similar trend is observed as in the transmitted pump power evolution. Discrepancy between predictions of the Eq. (21) and the measured gain is observed over the $P_{T h}$ [Fig. 5(b)]. However, the accuracies of Eq. (7) and Eq. (21) are very good agreement with all fiber lengths as soon as the input pump power is below $P_{T h}$.

\section{Conclusions}

In conclusion, highly accurate UPA based solution of pump, Stokes and Gain of BFA are introduced for the first time, to our best of knowledge, to the system of SBS equations in a lossy medium that

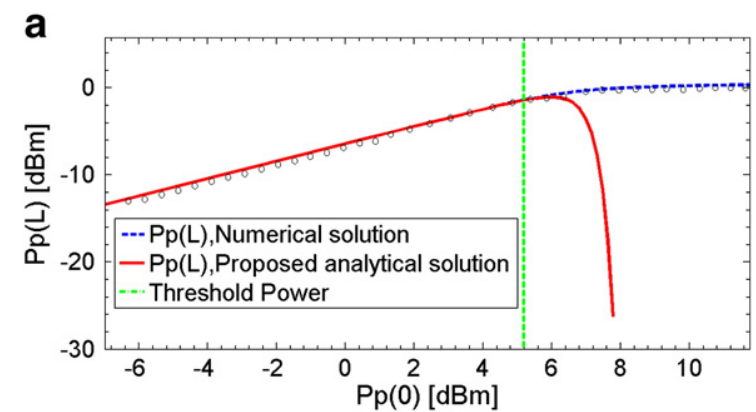

b

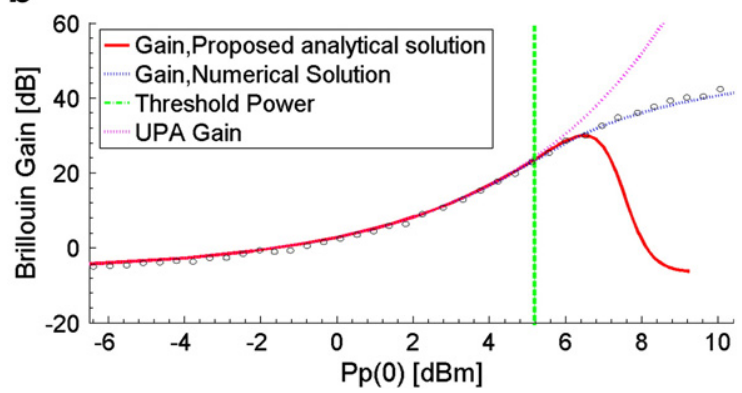

Fig. 5. (a) Transmitted pump power $P_{p}(L)$ and (b) BFA gain $\left(G_{B F A}\right)$ versus the input pump power $P_{p}(0)$. Open circles indicate experimental data. The same values are used as in Fig. 1. 
partly accounts for depletion. The validity range of the solution is defined by the accurate determination of the threshold power of the BFA. The obtained results are numerically and experimentally validated. Simulation results show that, at the worst case, the absolute error of the proposed solution is 5 and 2.3 times better than the UPA solution, for pump and Stokes and respectively. The result obtained can be used to optimize performance of moderate-gain BFAs which are widely used in microwave photonics, radio-over-fiber technology, and sensing.

\section{Acknowledgments}

The author acknowledges the financial support from the head of scientific research projects commission of Gazikent University.

\section{Appendix. Solution of Eq.(15)}

Eq. (15) is obtained with starting UPA solution of $I_{S}(z)$ in (A1) and can be solved as follows,

$$
\begin{aligned}
& I_{S}(z)=I_{S}(L) \cdot \exp (\alpha \cdot(L-z)) \\
& I_{S}(z)=I_{S}(L) \exp \left\{\alpha \cdot(z-L)+\int_{L}^{z} g_{B} I_{p}(0) \exp \left[g_{B} I_{S}(L) \frac{e^{-\alpha L}}{\alpha}\left(e^{\alpha \zeta}-1\right)-\alpha \zeta\right] d \zeta\right\}
\end{aligned}
$$

$$
\begin{aligned}
& \int_{L}^{z} g_{B} I_{p}(0) \exp \left[g_{B} I_{S}(L) \frac{e^{-\alpha L}}{\alpha}\left(e^{\alpha \zeta}-1\right)-\alpha \zeta\right] d \zeta= \\
& \left.=-I_{p}(0) \cdot\left(-\exp \left(g_{B} \cdot I_{S}(L) / \alpha\right)\right)+\exp \left(g_{B} \cdot I_{S}(L) \cdot \exp (\alpha \cdot L-\alpha \cdot z) / \alpha\right)\right) / I_{S}(L) \cdot C
\end{aligned}
$$

with,

$C=\exp \left(-\left(g_{B} \cdot I_{S}(L) \cdot \exp (\alpha \cdot L)+\alpha^{2} \cdot L\right) / \alpha\right)$

\section{References}

[1] N.A. Olsson, J.P. Van der Ziel, Appl. Phys. Lett. 48 (1986) 1329.

[2] R.W. Tkach, A.R. Chraplyvy, R.M. Derosier, IEEE Photon. Technol. Lett. 21 (1989) S105.

[3] K.-Y. Song, M. González-Herráez, L. Thévenaz, Opt. Express 13 (2005) 82.

[4] D. Culverhouse, F. Frahi, C.N. Pannell, D.A. Jackson, Electron. Lett. 25 (1989) 913.

[5] R.W. Boyd, Nonlinear Optics, 2nd edition Academic Press, New York, 2003 Chapter 9 .

[6] L. Chen, X. Bao, Opt. Commun. 152 (1998) 65.

[7] S. Le Floch, P. Cambon, J. Opt. Soc. Am. A 20 (2003) 1132.

[8] A. Kobyakov, S. Darmanyan, M. Sauer, D. Chowdhury, Opt. Lett. 31 (2006) 1960.

[9] Z. Ou, J. Li, L. Zhang, Z. Dai, Y. Liu, Opt. Commun. 282 (2009) 3812.

[10] F. Ravet, J. Snoddy, X. Bao, L. Chen, Open Opt. J. 2 (2008) 1.

[11] F. Ravet, X. Bao, L. Chen, Appl. Opt. 44 (2005) 5304. 